\title{
Comparison of the Metal Toxicity due to Aluminium and Barium on the Growth Attributes of Vigna trilobata (L.) Verde
}

\author{
A. Arundhathi, K. Marisamy, M. Duraipandian, R. Sevugaperumal, V. Ramasubramanian" \\ Department of Botany, Ayya Nadar Janaki Ammal College (Autonomous), India
}

Copyright $\bigcirc 2016$ by authors, all rights reserved. Authors agree that this article remains permanently open access under the terms of the Creative Commons Attribution License 4.0 International License

\begin{abstract}
The aim of this study was to investigate the ill effects of Aluminium and Barium on the morphological, biochemical and enzymatic characters of Vigna trilobata (L.) Verde which are commonly used in the fireworks industries of this area. After ten days treatment with different concentrations of aluminium and barium $(2,4,6,8$ \& $10 \mathrm{mM}$ ), the plant exhibited a decline in growth, chlorophyll contents, soluble sugar and protein than in the control. In contrary, the content of free amino acid, proline, leaf nitrate and the activities of enzymes such as catalase and peroxidase were found increased with the increase in the concentration of aluminium and barium while the activity of nitrate reductase were found to be decreased. Comparison of the parameters analyzed between the treated and control reveals that aluminium and barium have seriously affected the Vigna trilobata (L.) Verde. Based on this, it was concluded that high concentration of aluminium and barium severely affect the plant growth and result in growth inhibition, decline in physiological and biochemical activities of crop plants.
\end{abstract}

Keywords Barium, Aluminum, Free Amino Acid, Proline, Catalase and Peroxidase

\section{Introduction}

Exposure to heavy metal toxicity has become a major limiting factor in the growth of crop plants, affecting the sustainability of agricultural production [1]. Large parts of agricultural soil are contaminated with heavy metals by natural and anthropogenic activities. Heavy metals accumulated in excess in plant tissues, cause alteration in various physiological processes such as transpiration, photosynthesis and photosynthetic electron transport, biosynthesis of chlorophyll as well as cell membrane integrity [2]. Aluminium (Al) is not regarded as an essential nutrient, but at low concentrations sometimes increases plant growth or induces other desirable effects [3] [4]. Aluminium toxicity is a potential growth limiting factor for plants grown in acid soil in many part of the world [5]. Excess supply of aluminium causes phytotoxic effect [6]. Furthermore, it may cause indirect toxic effects by replacing essential nutrients at cation exchange sites in plants [7]. Barium is phytotoxic to plants at submillimolar (or) mllimolar levels and is thought to act through inhibition of $\mathrm{K}$ uptake by the roots. The various harmful effects of barium in cereals, include reduction in germination, root-shoot length, changes in the activity of various enzymes and poor grain yield [8]. The effects of barium on plant growth are lethal even as small concentrations can retard growth [9]. This study is aimed to find out the effect of various concentrations of aluminium and barium on Vigna trilobata (L.) Verde on the morphometric, pigmental, biochemical and enzymatic characteristics of Vigna trilobata (L.)Verde. In the present day context our study is very crucial because the soil in our area remains highly polluted due to aluminium and barium indiscriminately used in fireworks industries which effect most commonly cultivated Vigna trilobata (L.) Verde. - the chief crop of locals. Thus there is an urgent necessity to understand the ill effects of aluminium and barium on the crop plants mentioned and the necessary steps to be taken to overcome the problem.

\section{Material and Methods}

Both control and experimental plants were allowed to grow in soil garden soil. After seven days, the seedling of Vigna trilobata (L.)Verde. were treated with various concentrations of aluminium and bariumchloride such as 2, 4, $6,8 \& 10 \mathrm{mM}$ (mM - milimolar) individually. Ten days after that, the morphometric, pigmental, biochemical and enzymatic characters were analyzed.

The morphometric characters such as shoot length and root length were measured by scale and expressed in centimeter which is the unit of measurement length. The leaf area was measured by conventional graphical method by 
drawing the outline of the leaf in a graph sheet and counting the small and big squares within it and represented in $\mathrm{cm}^{2}$. The fresh weight and dry weight of the seedlings were found out using an electronic balance.

The pigmental characters such as chlorophyll a, chlorophyll b, total chlorophyll and carotenoids are estimated by conventional method [10].

Of the biochemical characteristics, the total soluble sugars was measured after centrifugation and further treating the tissue using tri-chloro acetic acid and anthrone as the indicator. The amino acid content was measured by homogenation of leaf tissue in ethanol using ninhydrine as the indicator [11]. The protein content was measured by centrifugation of ground leaf tissue in tri-chloro acetic acid, folin phenol with alkaline copper mixture [12]. The proline was estimated by centrifugation of fresh leaf tissue in sulphosalicylic acid, glacial acetic acid and finally separating with toluene [13].

The In vivo Nitrate Reductase (NR) activity was assayed according to Jaworski[14] method with modification. Fresh leaf material $(100 \mathrm{mg})$ was incubated in scintillation vials containing $5 \mathrm{ml}$ of incubation medium composed of, $100 \mathrm{mM}$ $\mathrm{KH}_{2} \mathrm{PO}_{4}-\mathrm{KOH}$ buffer $\mathrm{pH} 7.5,200 \mathrm{mM} \mathrm{KNO}_{3}, 1 \%(\mathrm{v} / \mathrm{v})$ n-Propanol and $0.1 \%(\mathrm{v} / \mathrm{v})$ Triton $\mathrm{X} 100$. Incubation was carried out in dark for one hour at room temperature with occasional shaking. Aliquots of $0.5 \mathrm{ml}$ was taken from the vials and analysed for nitrite formed after 1 hour incubation. To $0.5 \mathrm{ml}$ distilled water, $1 \mathrm{ml}$ of $3 \%$ sulphanilamide and 1 $\mathrm{ml}$ of N-1-N (Naphthyl ethylene-diamine dihydrochloride) were added in quick succession. Fifteen minutes were allowed for colour formation and absorbance was measured at $540 \mathrm{~nm}$. The nitrite was estimated with the help of a standard nitrite curve. The activities of peroxidase and catalase enzyme were estimated from, the leaves of experimental plants weighing about $1 \mathrm{~g}$ ground in $5 \mathrm{ml}$ of $100 \mathrm{mM}$ (mM - milimolar) phosphate buffer ( $\mathrm{pH}$ 6.0) filtered through a three layered cheese cloth and spun at 3000 rpm for 30 minutes. The supernatant obtained was served as the source for crude enzymes such as peroxidise and catalase.

To assay peroxidise activity, the enzyme extract was added to pyrogallol which gets oxidized to a coloured derivative in the presence of hydrogen peroxide $(1 \%(\mathrm{v} / \mathrm{v})$. The amount of purpurogallin formed during the reaction was assayed spectroscopically [15]. To $2 \mathrm{ml}$ of pyrogallol phosphate buffer $(0.058 \mathrm{M}$ pyrogallol dissolved in $0.1 \mathrm{M}$ phosphate buffer $\mathrm{pH} 6.0$ ), an aliquot of $0.1 \mathrm{ml}$ of enzyme extract was added. Then, absorbance was set to zero at $420 \mathrm{~nm}$. To this, $0.5 \mathrm{ml}$ of $\mathrm{H}_{2} \mathrm{O}_{2}(1 \%(\mathrm{v} / \mathrm{v}))$ was added. Then, the content was thoroughly mixed and the absorbance was measured using systronics model no. 106 spectrophotometer. The difference in the absorbance at an interval of 20 seconds for a period of 3 minutes was measured. The peroxidise activity was expressed as moles of $\mathrm{H}_{2} \mathrm{O}_{2}$ reduced per unit enzyme per unit time.

To assay the catalase activity, $3 \mathrm{ml}$ of phosphate buffer was added to $1 \mathrm{ml}$ of $\mathrm{H}_{2} \mathrm{O}_{2}$ and $1 \mathrm{ml}$ of enzyme extract [16]. The reaction mixture was incubated at $25^{\circ} \mathrm{C}$ for 1 minute. The reaction was terminated by the addition of $1 \mathrm{ml}$ of $\mathrm{H}_{2} \mathrm{SO}_{4}$. The reaction mixture was titrated against $0.01 \mathrm{~N}$ $\mathrm{KMNO}_{4}$. The end point was the persistence of pink colour at least for 15 seconds. The catalase activity was expressed in micromoles $\mathrm{H}_{2} \mathrm{O}_{2}$ catalysed.

\section{Statistics Analysis}

The growth parameters were determined with ten independent replicates. Biochemical characters and enzymatic assay were carried out for five times and the average was considered the data reported as mean $\pm \mathrm{SE}$ and with figures in parentheses represent the per cent activity. Statistical analysis (One way ANOVA - Tukey test) was applied using the statistical package, SPSS 16.0.

\section{Results}

Vigna trilobata (L.) Verde seedlings grown in different concentration of aluminium and barium exhibited inhibition in root and shoot length, leaf area, fresh weight, dry weight, with shoot being affected more than the root. After 10 days of treatment, a significant reduction of $55 \%$, and $32 \%$ and $63 \%, 34 \%$ in root growth and shoot growth respectively because of aluminium and barium was noticed under $10 \mathrm{Mm}$ concentration (Table 1). Similarly leaf area, fresh weight and dry weight also significantly decreased to tune of $25 \%$, $34 \%, 41 \%$, and $37 \%, 52 \%$, and $52 \%$ respectively at and $10 \mathrm{mM}$ concentration of aluminium and barium respectively. 
Table 1. Effect of various concentration of aluminium and barium on the morphometric charactristicofVigna trilobata (L.) Verde.(Black gram)

\begin{tabular}{|c|c|c|c|c|c|c|c|c|c|c|c|c|}
\hline \multirow{3}{*}{ S.NO } & \multirow{3}{*}{$\begin{array}{c}\text { Growth } \\
\text { parametre }\end{array}$} & \multicolumn{11}{|c|}{ Concentration of heavy metals } \\
\hline & & \multirow{2}{*}{ Control } & \multicolumn{2}{|c|}{$2 \mathrm{mM}$} & \multicolumn{2}{|c|}{$4 \mathrm{mM}$} & \multicolumn{2}{|c|}{$6 \mathrm{mM}$} & \multicolumn{2}{|c|}{$8 \mathrm{mM}$} & \multicolumn{2}{|c|}{$10 \mathrm{mM}$} \\
\hline & & & $\mathrm{Al}$ & $\mathrm{Ba}$ & $\mathrm{Al}$ & $\mathrm{Ba}$ & $\mathrm{Al}$ & $\mathrm{Ba}$ & $\mathrm{Al}$ & $\mathrm{Ba}$ & $\mathrm{Al}$ & $\mathrm{Ba}$ \\
\hline 1 & $\begin{array}{l}\text { Root length } \\
\quad(\mathrm{cm})\end{array}$ & $\begin{array}{c}9.62 \pm \\
0.01 \\
(100) \mathrm{a}^{*}\end{array}$ & $\begin{array}{c}8.67 \pm \\
0.05 \\
(96)\end{array}$ & $\begin{array}{c}8.1 \pm \\
0.11 \mathrm{a}^{*} \\
(93)\end{array}$ & $\begin{array}{c}7.71 \pm \\
0.11 \mathrm{a}^{*} \\
(90)\end{array}$ & $\begin{array}{c}7.5 \pm \\
0.01 a^{*} \\
(88)\end{array}$ & $\begin{array}{c}6.03 \pm \\
0.10 a^{*} \\
(82)\end{array}$ & $\begin{array}{c}5.98 \pm \\
0.02 \mathrm{a}^{*} \\
(80)\end{array}$ & $\begin{array}{c}3.57 \pm \\
0.04 \mathrm{a}^{*} \\
(53)\end{array}$ & $\begin{array}{c}3.01 \pm \\
0.05 \mathrm{a}^{*} \\
(46)\end{array}$ & $\begin{array}{c}2.98 \pm \\
0.02 \mathrm{a}^{*} \\
(45)\end{array}$ & $\begin{array}{c}3.84 \pm \\
0.32 \mathrm{a}^{*} \\
(37)\end{array}$ \\
\hline 2 & $\begin{array}{l}\text { Shoot length } \\
(\mathrm{cm})\end{array}$ & $\begin{array}{c}18.06 \pm \\
0.02 \\
(100)\end{array}$ & $\begin{array}{c}17.02 \pm \\
0.01 \mathrm{a}^{*} \\
(94)\end{array}$ & $\begin{array}{c}16.79 \pm \\
0.04 a^{*} \\
(90)\end{array}$ & $\begin{array}{c}16.79 \pm \\
0.28 \mathrm{a}^{*} \\
(93)\end{array}$ & $\begin{array}{c}16.02 \pm \\
0.08 \mathrm{a}^{*} \\
(89)\end{array}$ & $\begin{array}{c}16.3 \pm \\
0.14 a^{*} \\
(90)\end{array}$ & $\begin{array}{c}15.66 \pm \\
0.11 a^{*} \\
(87)\end{array}$ & $\begin{array}{c}15.67 \pm \\
0.01 \mathrm{a}^{*} \\
(87)\end{array}$ & $\begin{array}{c}12.01 \pm \\
0.01 a^{*} \\
(67)\end{array}$ & $\begin{array}{c}12.37 \pm \\
0.19 a^{*} \\
(68)\end{array}$ & $\begin{array}{c}11.37 \pm \\
0.10 \mathrm{a}^{*} \\
(66)\end{array}$ \\
\hline 3 & Leaf area & $\begin{array}{c}8.99 \pm \\
0.21 \\
(100) \\
\end{array}$ & $\begin{array}{c}7.91 \pm \\
0.09 \mathrm{a}^{*} \\
(89)\end{array}$ & $\begin{array}{c}6.88 \pm \\
0.01 \mathrm{a}^{*} \\
(84) \\
\end{array}$ & $\begin{array}{c}7.9 \pm \\
0.13 \mathrm{a}^{*} \\
(85) \\
\end{array}$ & $\begin{array}{c}7.81 \pm \\
0.31 \mathrm{a}^{*} \\
(80) \\
\end{array}$ & $\begin{array}{c}5.83 \pm \\
0.02 \mathrm{a}^{*} \\
(83) \\
\end{array}$ & $\begin{array}{c}4.97 \pm \\
0.01 a^{*} \\
(78) \\
\end{array}$ & $\begin{array}{c}5.29 \pm \\
0.11 \mathrm{a}^{*} \\
(76)\end{array}$ & $\begin{array}{c}3.11 \pm \\
0.01 \mathrm{a}^{*} \\
(69) \\
\end{array}$ & $\begin{array}{c}4.14 \pm \\
0.09 * \\
(75) \\
\end{array}$ & $\begin{array}{c}2.96 \pm \\
0.01 \mathrm{a}^{*} \\
(63) \\
\end{array}$ \\
\hline 4 & $\begin{array}{c}\text { Total fresh } \\
\text { weight }(\mathrm{gm})\end{array}$ & $\begin{array}{l}1.75 \pm \\
0.008 \\
(100) \\
\end{array}$ & $\begin{array}{c}1.53 \pm \\
0.01 \mathrm{a}^{*} \\
(88)\end{array}$ & $\begin{array}{c}1.49 \pm \\
0.01 \mathrm{a}^{*} \\
(74)\end{array}$ & $\begin{array}{c}1.45 \pm \\
0.07 \mathrm{a}^{*} \\
(83) \\
\end{array}$ & $\begin{array}{c}1.41 \pm \\
0.07 \mathrm{a}^{*} \\
(68)\end{array}$ & $\begin{array}{c}1.27 \pm \\
0.02 \mathrm{a}^{*} \\
(70)\end{array}$ & $\begin{array}{c}1.33 \pm \\
0.09 \mathrm{a}^{*} \\
(54)\end{array}$ & $\begin{array}{c}1.27 \pm \\
0.02 \mathrm{a}^{*} \\
(69)\end{array}$ & $\begin{array}{c}1.001 \pm \\
0.02 \mathrm{a}^{*} \\
(50)\end{array}$ & $\begin{array}{c}1.15 \pm \\
0.02 \mathrm{a}^{*} \\
(66)\end{array}$ & $\begin{array}{c}0.998 \pm \\
0.11 \mathrm{a}^{*} \\
(48) \\
\end{array}$ \\
\hline 5 & $\begin{array}{c}\text { Total dry } \\
\text { weight (gm) }\end{array}$ & $\begin{array}{c}0.308 \pm \\
0.1 \\
(100)\end{array}$ & $\begin{array}{c}0.236 \pm \\
0.01 \mathrm{a}^{*} \\
(77)\end{array}$ & $\begin{array}{c}0.211 \pm \\
0.01 a^{*} \\
1(71)\end{array}$ & $\begin{array}{c}0.212 \pm \\
0.01 \mathrm{a}^{*} \\
(68)\end{array}$ & $\begin{array}{c}0.20 \pm \\
0.02 * \\
(64)\end{array}$ & $\begin{array}{c}0.191 \pm \\
0.01 a^{*} \\
(62)\end{array}$ & $\begin{array}{c}0.190 \pm \\
0.03 \mathrm{a}^{*} \\
(61)\end{array}$ & $\begin{array}{c}0.191 \pm \\
0.04 \mathrm{a}^{*} \\
(62)\end{array}$ & $\begin{array}{c}0.167 \pm \\
0.01 a^{*} \\
(53)\end{array}$ & $\begin{array}{c}0.185 \pm \\
0.07 \mathrm{a}^{*} \\
(59)\end{array}$ & $\begin{array}{c}0.181 \pm 0 \\
.05 \mathrm{a}^{*} \\
(48)\end{array}$ \\
\hline
\end{tabular}

Values in parameters indicate percent activity; values are represents means of five observation. Values in parentheses are activity with respect to control. Mean $( \pm)$ SE. a - refers to values compared with control in various concentrations of metals, $\mathrm{a}^{*}$ - refers to ignificant $(\mathrm{P} \leq 0.05$ - Tukey test). a\# - refers to non - significant. Al - Aluminium; Ba - Barium; mM - Milimolar; cm- centimeter

The effect of different concentration of aluminium and barium on photosynthetic pigments is depicted in Table. 2 . Photosynthetic pigments were significantly decreased with increasing level of aluminium and barium. The reduction of chlorophyll $a, b$, total chlorophyll and carotenoids was $52 \%, 58 \%, 45 \%$ and $61 \%$ and $56 \%, 61 \%, 64 \%$, and $73 \%$ respectively at $10 \mathrm{mM}$ aluminium and barium treatment compared to the control. Aluminium and barium also modified the total soluble sugar and protein content in Vigna trilobata (L.) Verde leaves (Table. 3). Under the $10 \mathrm{mM}$ aluminium and barium concentration the decrease in the total soluble sugar and protein was $53 \%$ and $31 \%$ and $59 \% 40 \%$ respectively. However the content of the free amino acids, proline and leaf nitrate significantly increased with increasing concentration of aluminium and barium.

Table 2. Effect of various concentrations of aluminium and barium on the pigments content of Vigna trilobata (L.) Verde (Black gram)

\begin{tabular}{|c|c|c|c|c|c|c|c|c|c|c|c|c|}
\hline \multirow{3}{*}{$\begin{array}{l}\text { S. } \\
\text { NO }\end{array}$} & \multirow{3}{*}{$\begin{array}{c}\text { Growth } \\
\text { parametre }\end{array}$} & \multicolumn{11}{|c|}{ Concentration of heavy metals } \\
\hline & & \multirow[b]{2}{*}{ Control } & \multicolumn{2}{|c|}{$2 \mathrm{mM}$} & \multicolumn{2}{|c|}{$4 \mathrm{mM}$} & \multicolumn{2}{|c|}{$6 \mathrm{mM}$} & \multicolumn{2}{|c|}{$8 \mathrm{mM}$} & \multicolumn{2}{|c|}{$10 \mathrm{mM}$} \\
\hline & & & $\mathrm{Al}$ & $\mathrm{Ba}$ & $\mathrm{Al}$ & $\mathrm{Ba}$ & $\mathrm{Al}$ & $\mathrm{Ba}$ & $\mathrm{Al}$ & $\mathrm{Ba}$ & $\mathrm{Al}$ & $\mathrm{Ba}$ \\
\hline 1 & $\begin{array}{c}\text { Chlorophyll a } \\
\text { (mg/g LFW) }\end{array}$ & $\begin{array}{c}3.36 \pm \\
0.01 \\
(100) \\
\end{array}$ & $\begin{array}{c}3.19 \pm \\
0.1 \mathrm{a}^{*} \\
(93) \\
\end{array}$ & $\begin{array}{c}2.92 \pm \\
0.03 * \\
(88) \\
\end{array}$ & $\begin{array}{c}2.49 \pm \\
0.01 \mathrm{a}^{*} \\
(78) \\
\end{array}$ & $\begin{array}{c}2.41 \pm 0 \\
.02 \mathrm{a}^{*} \\
(73) \\
\end{array}$ & $\begin{array}{c}2.30 \pm \\
0.13 a^{*} \\
(65) \\
\end{array}$ & $\begin{array}{c}2.21 \pm \\
0.11 \mathrm{a}^{*} \\
(60) \\
\end{array}$ & $\begin{array}{c}2.17 \pm \\
0.3 \mathrm{a}^{*} \\
(55) \\
\end{array}$ & $\begin{array}{c}1.98 \pm \\
0.01 \mathrm{a}^{*} \\
(52) \\
\end{array}$ & $\begin{array}{c}1.94 \pm \\
0.03 \mathrm{a}^{*} \\
(48) \\
\end{array}$ & $\begin{array}{c}1.11 \pm \\
0.05 \mathrm{a}^{*} \\
(44) \\
\end{array}$ \\
\hline 2 & $\begin{array}{l}\text { Chlorophyllb } \\
\text { (mg/g LFW) }\end{array}$ & $\begin{array}{c}3.98 \pm \\
0.1 \\
(100)\end{array}$ & $\begin{array}{c}2.67 \pm \\
0.31 \\
a^{*} \\
(94)\end{array}$ & $\begin{array}{c}2.36 \pm \\
0.31 \mathrm{a}^{*} \\
(91)\end{array}$ & $\begin{array}{c}2.44 \pm 0.0 \\
5 \mathrm{a}^{*} \\
(71)\end{array}$ & $\begin{array}{c}2.15 \pm \\
0.05 \mathrm{a}^{*} \\
(78)\end{array}$ & $\begin{array}{c}2.31 \pm \\
0.21 \mathrm{a}^{*} \\
(62)\end{array}$ & $\begin{array}{c}2.0 \pm 0 \\
03 \mathrm{a}^{*} \\
(66)\end{array}$ & $\begin{array}{c}1.99 \pm \\
0.03 \mathrm{a}^{*} \\
(52)\end{array}$ & $\begin{array}{c}1.53 \pm 0 . \\
09 \mathrm{a}^{*} \\
(47)\end{array}$ & $\begin{array}{c}1.63 \pm \\
0.01 \mathrm{a}^{*} \\
(42)\end{array}$ & $\begin{array}{c}1.18 \pm 0 . \\
1 \mathrm{a}^{*} \\
(39)\end{array}$ \\
\hline 3 & $\begin{array}{c}\text { Total } \\
\text { Chlorophyll } \\
\text { content } \\
(\mathrm{mg} / \mathrm{g} \text { LFW) }\end{array}$ & $\begin{array}{l}5.34 \pm \\
0.081 \\
(100)\end{array}$ & $\begin{array}{c}4.633 \\
\pm \\
0.11 \\
a^{*} \\
(79)\end{array}$ & $\begin{array}{c}4.6 \pm \\
0.08 \mathrm{a}^{*} \\
(78)\end{array}$ & $\begin{array}{c}4.44 \pm \\
0.04 \mathrm{a}^{*} \\
(73)\end{array}$ & $\begin{array}{c}4.39 \pm \\
0.02 \mathrm{a}^{*} \\
(69)\end{array}$ & $\begin{array}{c}4.15 \pm \\
0.12 \mathrm{a}^{*} \\
(64)\end{array}$ & $\begin{array}{c}4.17 \pm \\
0.01 \mathrm{a}^{*} \\
(65)\end{array}$ & $\begin{array}{c}3.94 \pm \\
0.06 \mathrm{a}^{*} \\
(58)\end{array}$ & $\begin{array}{c}3.52 \pm 0 . \\
08 \mathrm{a}^{*} \\
(49)\end{array}$ & $\begin{array}{c}3.83 \pm 0 . \\
11 \mathrm{a}^{*} \\
(55)\end{array}$ & $\begin{array}{c}3.113 \pm \\
0.01 \mathrm{a}^{*} \\
(34)\end{array}$ \\
\hline 4 & $\begin{array}{c}\text { Total } \\
\text { carotinoid } \\
\text { content } \\
\text { (mg/g LFW) }\end{array}$ & $\begin{array}{c}1.71 \pm \\
0.02 \\
(100)\end{array}$ & $\begin{array}{c}1.59 \pm \\
0.01 \\
a^{*} \\
(85)\end{array}$ & $\begin{array}{c}1.51 \pm 0 . \\
01 \mathrm{a}^{*} \\
(80)\end{array}$ & $\begin{array}{c}1.47 \pm \\
0.02 \mathrm{a}^{*} \\
(73)\end{array}$ & $\begin{array}{c}1.43 \pm \\
0.02 \mathrm{a}^{*} \\
(71)\end{array}$ & $\begin{array}{c}1.35 \pm 0 . \\
01 \mathrm{a}^{*} \\
(57)\end{array}$ & $\begin{array}{c}1.3 \pm \\
0.01 \mathrm{a}^{*} \\
(53)\end{array}$ & $\begin{array}{c}1.26 \pm 0 \\
01 \mathrm{a}^{*} \\
(46)\end{array}$ & $\begin{array}{c}1.21 \pm 0 \\
01 \mathrm{a}^{*} \\
(40)\end{array}$ & $\begin{array}{c}1.17 \pm 0 . \\
02 \mathrm{a}^{*} \\
(39)\end{array}$ & $\begin{array}{c}1.08 \pm \\
0.02 \mathrm{a}^{*} \\
(27)\end{array}$ \\
\hline
\end{tabular}

Values in parameters indicate percent activity; values are represents means of five observation. Values in parentheses are activity with respect to control. Mean $( \pm)$ SE. a - refers to values compared with control in various concentrations of metals, $\mathrm{a}^{*}$ - refers to ignificant ( $\mathrm{P} \leq 0.05$ - Tukey test). a\# - refers to non - significant. Al - Aluminium; Ba - Barium; mM - Milimolar; Al - Aluminium; Ba - Barium; mM - Milimolar; cm- centimeter; mg - milligram; ggram; LFW - Leaf fresh weight 
Table 3. Effect of various concentrations of aluminium and barium on the biochemical characteristic of Vigna trilobata (L.) Verde (Black gram)

\begin{tabular}{|c|c|c|c|c|c|c|c|c|c|c|c|c|}
\hline \multirow{3}{*}{$\begin{array}{l}\text { S. } \\
\text { NO }\end{array}$} & \multirow{3}{*}{$\begin{array}{c}\text { Growth } \\
\text { parametre }\end{array}$} & \multicolumn{11}{|c|}{ Concentration of heavy metals } \\
\hline & & \multirow[b]{2}{*}{ Control } & \multicolumn{2}{|c|}{$2 \mathrm{mM}$} & \multicolumn{2}{|c|}{$4 \mathrm{mM}$} & \multicolumn{2}{|c|}{$6 \mathrm{mM}$} & \multicolumn{2}{|c|}{$8 \mathrm{mM}$} & \multicolumn{2}{|c|}{$10 \mathrm{mM}$} \\
\hline & & & $\mathrm{Al}$ & $\mathrm{Ba}$ & $\mathrm{Al}$ & $\mathrm{Ba}$ & $\mathrm{Al}$ & $\mathrm{Ba}$ & $\mathrm{Al}$ & $\mathrm{Ba}$ & $\mathrm{Al}$ & $\mathrm{Ba}$ \\
\hline 1 & $\begin{array}{l}\text { Total soluble } \\
\text { sugar } \\
(\mathrm{mg} / \mathrm{g} \mathrm{LFW})\end{array}$ & $\begin{array}{c}6.46 \pm \\
0.1 \\
(100) \\
\end{array}$ & $\begin{array}{c}5.94 \pm 0 \\
.01 \mathrm{a}^{*} \\
(92) \\
\end{array}$ & $\begin{array}{c}5.89 \pm \\
0.1 \mathrm{a}^{*} \\
(89) \\
\end{array}$ & $\begin{array}{c}5.82 \pm \\
0.03 \mathrm{a}^{*} \\
(80)\end{array}$ & $\begin{array}{c}5.76 \pm 0 \\
.09 \mathrm{a}^{*} \\
(72) \\
\end{array}$ & $\begin{array}{c}5.15 \pm \\
0.21 \mathrm{a}^{*} \\
(68)\end{array}$ & $\begin{array}{c}5.15 \pm \\
0.3 \mathrm{a}^{*} \\
(68)\end{array}$ & $\begin{array}{c}4.9 \pm \\
0.05 \mathrm{a}^{*} \\
(58)\end{array}$ & $\begin{array}{c}4.68 \pm \\
0.05 \mathrm{a}^{*} \\
(50)\end{array}$ & $\begin{array}{c}4.37 \pm \\
0.31 \mathrm{a}^{*} \\
(47)\end{array}$ & $\begin{array}{c}4.13 \pm \\
0.31 \mathrm{a}^{*} \\
(41)\end{array}$ \\
\hline 2 & $\begin{array}{c}\text { Total soluble } \\
\text { protein }(\mathrm{mg} / \mathrm{g} \\
\mathrm{LFW})\end{array}$ & $\begin{array}{c}27.79 \pm \\
0.1 \\
(100) \\
\end{array}$ & $\begin{array}{c}27.21 \pm \\
0.41 \mathrm{a}^{*} \\
(95) \\
\end{array}$ & $\begin{array}{c}26.87 \pm \\
0.4 \mathrm{a}^{*} \\
(90) \\
\end{array}$ & $\begin{array}{c}26.63 \pm \\
0.21 \mathrm{a}^{*} \\
(92) \\
\end{array}$ & $\begin{array}{c}24.91 \pm \\
0.11 \mathrm{a}^{*} \\
(81) \\
\end{array}$ & $\begin{array}{c}25.43 \pm \\
0.14 \mathrm{a}^{*} \\
(85) \\
\end{array}$ & $\begin{array}{c}21.88 \pm \\
0.16 \\
a^{*}(70) \\
\end{array}$ & $\begin{array}{c}22.78 \pm 0 \\
.01 \mathrm{a}^{*} \\
(79) \\
\end{array}$ & $\begin{array}{c}21.03 \pm 0 \\
.21 \mathrm{a}^{*} \\
(65) \\
\end{array}$ & $\begin{array}{c}21.23 \pm \\
0.02 \mathrm{a}^{*} \\
(69) \\
\end{array}$ & $\begin{array}{c}20.65 \pm \\
0.01 \mathrm{a}^{*} \\
(60)\end{array}$ \\
\hline 3 & $\begin{array}{c}\text { Total free } \\
\text { amino acid } \\
(\mathrm{mg} / \mathrm{g} \text { LFW })\end{array}$ & $\begin{array}{c}0.34 \pm \\
0.1 \\
(100) \\
\end{array}$ & $\begin{array}{c}0.98 \pm \\
0.3 \mathrm{a}^{*} \\
(165) \\
\end{array}$ & $\begin{array}{c}0.81 \pm \\
0.04 \mathrm{a}^{*} \\
(156) \\
\end{array}$ & $\begin{array}{c}1.74 \pm \\
0.02 \mathrm{a}^{*} \\
(256) \\
\end{array}$ & $\begin{array}{l}1.70 \pm \\
0.1 \mathrm{a}^{*} \\
(250) \\
\end{array}$ & $\begin{array}{c}2.45 \pm \\
0.16 a^{*} \\
(321) \\
\end{array}$ & $\begin{array}{l}2.14 \pm \\
0.1 \mathrm{a}^{*} \\
(318) \\
\end{array}$ & $\begin{array}{c}2.99 \pm \\
0.01 \mathrm{a}^{*} \\
(385) \\
\end{array}$ & $\begin{array}{c}2.92 \pm 0 \\
31 \mathrm{a}^{*} \\
(380)\end{array}$ & $\begin{array}{l}4.01 \pm 0 \\
.02 \mathrm{a}^{*} \\
(410) \\
\end{array}$ & $\begin{array}{l}2.99 \pm \\
0.1 \mathrm{a}^{*} \\
(390) \\
\end{array}$ \\
\hline 4 & $\begin{array}{c}\text { Proline } \\
\text { content }(\mathrm{mg} / \mathrm{g} \\
\text { LFW) }\end{array}$ & $\begin{array}{c}0.9 \pm \\
0.10 \\
(100) \\
\end{array}$ & $\begin{array}{c}0.992 \pm \\
0.1 \mathrm{a}^{*} \\
(155) \\
\end{array}$ & $\begin{array}{l}1.11 \pm \\
0.1 \mathrm{a}^{*} \\
(166) \\
\end{array}$ & $\begin{array}{c}1.451 \pm \\
0.13 \mathrm{a}^{*} \\
(213) \\
\end{array}$ & $\begin{array}{c}1.921 \pm \\
0.11 \mathrm{a}^{*} \\
(210) \\
\end{array}$ & $\begin{array}{l}2.43 \pm 0 \\
.1 \quad a^{*} \\
(290) \\
\end{array}$ & $\begin{array}{c}2.39 \pm 0 \\
.14 \mathrm{a}^{*} \\
(285) \\
\end{array}$ & $\begin{array}{c}3.03 \pm 0 . \\
31 \mathrm{a}^{*} \\
(364) \\
\end{array}$ & $\begin{array}{c}2.534 \pm 0 \\
.11 \mathrm{a}^{*} \\
(356) \\
\end{array}$ & $\begin{array}{c}3.13 \pm 0 \\
.2 \mathrm{a}^{*} \\
(385) \\
\end{array}$ & $\begin{array}{c}3.11 \pm 0 . \\
13 \mathrm{a}^{*} \\
(380)\end{array}$ \\
\hline 5 & $\begin{array}{l}\text { Leaf nitrate } \\
(\mathrm{mg} / \mathrm{g} \text { LWF) }\end{array}$ & $\begin{array}{c}1.34 \pm \\
0.10 \\
(100) \\
\end{array}$ & $\begin{array}{c}2.73 \pm \\
0.04 a^{*} \\
(145) \\
\end{array}$ & $\begin{array}{c}2.87 \pm 0 \\
.19 \mathrm{a}^{*} \\
(156) \\
\end{array}$ & $\begin{array}{c}3.11 \pm \\
0.02 \mathrm{a}^{*} \\
(228) \\
\end{array}$ & $\begin{array}{c}3.09 \pm \\
0.02 \mathrm{a}^{*} \\
(212) \\
\end{array}$ & $\begin{array}{l}3.16 \pm \\
0.4 a^{*} \\
(275) \\
\end{array}$ & $\begin{array}{c}3.45 \pm 0 \\
.1 \mathrm{a}^{*} \\
(258) \\
\end{array}$ & $\begin{array}{c}3.59 \pm \\
0.04 \mathrm{a}^{*} \\
(364) \\
\end{array}$ & $\begin{array}{c}4.04 \pm \\
0.09 \mathrm{a}^{*} \\
(393)\end{array}$ & $\begin{array}{l}4.98 \pm \\
0.3 \mathrm{a} * \\
(401)\end{array}$ & $\begin{array}{c}4.34 \pm \\
0.02 \mathrm{a}^{*} \\
(411)\end{array}$ \\
\hline
\end{tabular}

Values in parameters indicate percent activity; values are represents means of five observation. Values in parentheses are activity with respect to control. Mean $( \pm)$ SE. a - refers to values compared with control in various concentrations of metals, $\mathrm{a}^{*}$ - refers to ignificant $(\mathrm{P} \leq 0.05$ - Tukey test). a\# - refers to non - significant. Al - Aluminium; Ba - Barium; mM - Milimolar; Al - Aluminium; Ba - Barium; mM - Milimolar; cm- centimeter; mg - milligram; ggram; LFW - Leaf fresh weight

The result of the present study shows that, in vivo NR activity of the leaves was significantly inhibited to $24 \%, 26 \%$ at $10 \mathrm{mM}$ concentration of aluminium and barium. There was a tremendous increase rise in catalase and peroxidase activity with significant increase in different aluminium and barium treatments (Table. 4). The increase was about 190\%, 175\%; 31\%, 33 at $6 \mathrm{mM}$ respectively.

Table 4. Effect of various concentrations of aluminium and barium on the enzyme activites of Vigna trilobata (L .) Verde (Black gram)

\begin{tabular}{|c|c|c|c|c|c|c|c|c|c|c|c|c|}
\hline \multirow{3}{*}{$\begin{array}{l}\text { S. } \\
\text { NO }\end{array}$} & \multirow{3}{*}{$\begin{array}{l}\text { Growth } \\
\text { parametre }\end{array}$} & \multicolumn{11}{|c|}{ Concentration of heavy metals } \\
\hline & & \multirow{2}{*}{ Control } & \multicolumn{2}{|c|}{$2 \mathrm{mM}$} & \multicolumn{2}{|c|}{$4 \mathrm{mM}$} & \multicolumn{2}{|c|}{$6 \mathrm{mM}$} & \multicolumn{2}{|c|}{$8 \mathrm{mM}$} & \multicolumn{2}{|c|}{$10 \mathrm{mM}$} \\
\hline & & & $\mathrm{Al}$ & $\mathrm{Ba}$ & $\mathrm{Al}$ & $\mathrm{Ba}$ & $\mathrm{Al}$ & $\mathrm{Ba}$ & $\mathrm{Al}$ & $\mathrm{Ba}$ & $\mathrm{Al}$ & $\mathrm{Ba}$ \\
\hline 1 & $\begin{array}{c}\text { In vivo NR } \\
\text { activity } \\
(\mu \text { mole } / \mathrm{g} \\
\mathrm{FW})\end{array}$ & $\begin{array}{c}24.16 \pm \\
0.05 \\
(100)\end{array}$ & $\begin{array}{c}23.25 \pm \\
0.1 \mathrm{a}^{*} \\
(90)\end{array}$ & $\begin{array}{c}22.01 \pm \\
0.13 \mathrm{a}^{*} \\
(87)\end{array}$ & $\begin{array}{c}21.49 \pm \\
0.11 \mathrm{a}^{*} \\
(80)\end{array}$ & $\begin{array}{c}21.99 \pm 0 \\
21 \mathrm{a}^{*} \\
(87)\end{array}$ & $\begin{array}{c}19.96 \pm \\
0.05 \mathrm{a}^{*} \\
(87)\end{array}$ & $\begin{array}{c}19.99 \pm \\
0.13 \mathrm{a} * \\
(79)\end{array}$ & $\begin{array}{c}18.31 \pm \\
0.05 \mathrm{a}^{*} \\
(77)\end{array}$ & $\begin{array}{c}18.94 \pm 0 \\
.01 \mathrm{a}^{*} \\
(75)\end{array}$ & $\begin{array}{c}17.46 \pm \\
0.11 \mathrm{a}^{*} \\
(76)\end{array}$ & $\begin{array}{c}17.46 \pm \\
0.1 \mathrm{a}^{*} \\
(74)\end{array}$ \\
\hline 2 & $\begin{array}{c}\text { Catalase } \\
\text { activity } \\
(\mu \text { mole/g } \\
\text { FW })\end{array}$ & $\begin{array}{c}0.013 \pm \\
0.1 \\
(100)\end{array}$ & $\begin{array}{c}0.094 \pm \\
0.1 \mathrm{a}^{*} \\
(183)\end{array}$ & $\begin{array}{c}0.09 \pm 0 . \\
3 \mathrm{a}^{*} \\
(190)\end{array}$ & $\begin{array}{c}0.162 \pm 0 \\
.02 \mathrm{a}^{*} \\
(250)\end{array}$ & $\begin{array}{c}0.173 \pm 0 . \\
01 \mathrm{a}^{*} \\
(263)\end{array}$ & $\begin{array}{c}0.288 \pm \\
0.13 a^{*} \\
(290)\end{array}$ & $\begin{array}{l}0.22 \pm \\
0.2 \mathrm{a}^{*} \\
(275)\end{array}$ & $\begin{array}{c}0.3 \pm \\
0.17 a^{*} \\
(365)\end{array}$ & $\begin{array}{c}0.37 \pm \\
0.11 \mathrm{a}^{*} \\
(388)\end{array}$ & $\begin{array}{c}0.411 \pm \\
0.01 \mathrm{a}^{*} \\
(401)\end{array}$ & $\begin{array}{c}0.39 \pm \\
0.03 \mathrm{a}^{*} \\
(381)\end{array}$ \\
\hline 3 & $\begin{array}{l}\text { Peroxidase } \\
\text { activity ( } \mu \\
\text { mole/g FW) }\end{array}$ & $\begin{array}{l}1.83 \pm \\
0.013 \\
(100)\end{array}$ & $\begin{array}{c}5.01 \pm \\
0.24 \mathrm{a}^{*} \\
(123)\end{array}$ & $\begin{array}{c}5.5 \pm \\
0.04 \mathrm{a}^{*} \\
(125)\end{array}$ & $\begin{array}{c}6.18 \pm \\
0.04 \mathrm{a}^{*} \\
(128)\end{array}$ & $\begin{array}{c}7.18 \pm \\
0.14 \mathrm{a}^{*} \\
(129)\end{array}$ & $\begin{array}{c}11.25 \pm \\
0.01 a^{*} \\
(131)\end{array}$ & $\begin{array}{c}14.25 \pm \\
0.02 \mathrm{a}^{*} \\
(133)\end{array}$ & $\begin{array}{c}17.31 \pm \\
0.01 \mathrm{a}^{*} \\
(138)\end{array}$ & $\begin{array}{c}15.3 \pm \\
0.02 \mathrm{a}^{*} \\
(134)\end{array}$ & $\begin{array}{c}19.44 \pm \\
0.11 \mathrm{a}^{*} \\
(141)\end{array}$ & $\begin{array}{c}16.44 \pm \\
0.1 \mathrm{a}^{*} \\
(136)\end{array}$ \\
\hline
\end{tabular}

Values in parameters indicate percent activity; values are represents means of five observation. Values in parentheses are activity with respect to control. Mean ( \pm ) SE. a - refers to values compared with control in various concentrations of metals, $a^{*}$ - refers to ignificant ( $\mathrm{P} \leq 0.05$ - Tukey test). a\# - refers to non - significant. Al - Aluminium; Ba - Barium; mM - Milimolar; Al - Aluminium; Ba - Barium; mM - Milimolar; cm- centimeter; mg - milligram; ggram; LFW - Leaf fresh weight

\section{Discussion}

In the present study, the different concentration of aluminium and barium caused a significant reduction in root length, shoot length, leaf area, fresh weight and dry weight of the plant with increased metal concentration. Similar report was observed by Minakshiet al. [17] in Ocimum tenuifolium under heavy stress. Sevugaperumal et al. [18] reported that higher concentration of aluminium reduced the root length, shoot length, leaf area, fresh weight and dry weight in Vigna radiata $\mathrm{L}$. falling in the line with the findings of Mahapatra, et al. [19] in Vigna radiata L. According to Shafiq et al. [20] reduced root length is due to reductions in both new cell formation and cell elongation in the extension region of the root. The reduction in plant growth during stress is due to low water potential, hampered nutrient uptake and secondary stress such as oxidative stress [21].

The photosynthetic process is related to biomass accumulation, which in turn, relies upon the pigment level. The chlorophyll content, which is an indicator of the photosynthetic activity of plants, showed a remarkable reduction in aluminium and barium treated plants. The decrease in these plant pigments may be due to cellular disorganization under heavy metal toxicity which causes agglutination of chloroplast [22]. Reduced Leaf chlorophyll and carotenoid contents with the application of aluminium 
and barium in the present study is in accordance with the earlier reports of Boddi et al. [23][24][25] [26]. The decrease in chlorophyll content was also reported in sunflower [27] and in almond [28].

The decrease in total soluble sugar content coincides with the photosynthetic inhibition or stimulation of respiration rate [29]. Sarma et al. [30] reported that the reduction of sugar content may be the result of the minimum uptake of phosphate. Reduction in protein level observed in the present study may be attributed to the decrease in protein synthesis or due to the denaturation of the enzymes involved in the synthesis of proteins [31].The reduction in soluble protein level with associated increase in free amino acids under stress may be attributed both to the disruption in protein synthesis [32] as well as to the enhanced proteolysis [33]. Reduced protein content may be due to either inhibition of protein synthesis by Orcutt and Nilsen, [34] or unavailability of essential component aminoacids [35] or inhibition of aminoacids mobilization to the site of protein synthesis [36]. There was a linear increase in free proline accumulation with increasing metal concentrations in the leaf of Abelmoschus, Brassica and Amaranthus. Proline accumulation is considered as an adaptive response to stress [37]. Accumulation of proline has frequently been used as a biochemical marker for water stress in plants [38]. Proline accumulation is considered a protective device for the plants to preserve water, which is necessary to tide over any internal water deficit situation [39]. The leaf nitrate content was analysed and found to be more in all the heavy metal treated plants. The accumulation of leaf nitrate content was found to be associated with a reduction in in vivo nitrate reductase (NR) activity. Similar increase in leaf nitrate content, reduction in in vivo NR activities with increased in concentration of cadmium chloride on Vigna radiata has been reported earlier [40]. The increased peroxidase activity caused a major impact on the chlorophyll degradation [41]. Catalase is another antioxidant scavenging enzyme. Catalase is special type of peroxidative enzyme which catalyses the degradation of $\mathrm{H}_{2} \mathrm{O}_{2}$ which is a natural metabolite toxic to plants [42]. The mechanism involved for reducing toxicity may be due to chelating of the metal ions by the ligands, which include organic acids, amino acids, peptides or polypeptides [43].

\section{Conclusions}

Conclusively, our results show that aluminium and barium at high concentration exceeding $2 \mathrm{mM}$ decreased the growth, chlorophyll content, protein and sugar content but increased the content of proline, free amino acid \& leaf nitrate. Our result suggest that the presence of aluminium and barium in low concentration is good for Vigna trilobata (L.) Verde growth but in high concentration make the soil toxic to the crop plants and result in growth inhibition, structural damage, decline in physiological and biochemical activities of crop plants.

\section{Acknowledgement}

The author is very much thankful to Dr. V. Ramasubramanian, Associate Professor and Head Department of Plant Biology and Plant Biotechnology, ANJA College, Sivakasi.Dr.R. Sevugaperumal, Associate Professor of Botany, ANJA College, Sivakasi for their guidance and The management of ANJA College, Sivakasi for providing necessary facilities to carry out the present study.

\section{REFERENCES}

[1] Lin, S.C., Ye, G.W., Wu, J.Z., Zheng, Y.B. and Lin, W.X. (2005). Studies on the Comparison of the Chemical Compounds in Lotus Seeds (Nelumbonucifera Gaertn.) Between "Tai-Kong lotus 36" and "Jian lotus". Strait Pharmaceutical Journal., 17(4): 91-93.

[2] Hussain, K., Sahadevan, K.K. and Salim, N. (2010). Bioaccumulation and release of mercury in Vigna mungo (L.) Hepper seedlings. Journal of stress physiology \& Biochemistry., 6(3): 56-63.

[3] Foy, C.D. and Fleming, A.L. (1982) Aluminium tolerance of two wheat cultivars related to nitrate reductase activities. J. Plant. Nutr., 5: 1313-1333.

[4] Foy, C.D. (1983). The physiology of plant adaptation to metal stress. Iowa State J. Res., 57: 355-391.

[5] Foy, C.D. (1996). Tolerance of Barley cultivars to an acid, aluminium-toxic subsoil related to mineral element concentrations of their shoots. J. Plant Nutr., 19: 1361-1380.

[6] Juste, C. and Mench, M. (1992). Long term application of sewage sludge and its effect on metal uptake by crops. In: D.C. Adriano (Ed), Biogeochemistry of trace metals, Ann.Arbor, London, Tokyo, Leuwis publishers. 159-193.

[7] Taiz, L. and Zeiger, E.S. (2002). Plant physiology ( $3^{\text {rd }}$ eds.). Sinauer Associations., M.A. Sundarland. 690.

[8] Suwa, R.K., Jayachandran, N.T., Nguyen, A., Boulenouar, K., Fujita, H. and Saneoka. (2008). Barium Toxicity Effects in Soybean Plants. Archives of Environmental Contamination and Toxicology., 55(3): 397-403.

[9] Robinson, W.O., Whetstone. R, R. and Byers, H.G. (1938) Studies on infertile soils II. Soil high in barium. Soil Sci. Soc. Amer. Proc., 3: 87-89.

[10] Wellburn, A.R. and Lichtenthaler, H. (1984). In Advances in photosynthesis Research, (ed. Sybesma) Martinus Nijhoff, Co. The Hague. II: 9-12.

[11] Jayaraman, J. (1981). Laboratory manual in Biochemistry, Willey-Eastern Company Limited, Madras. 1 -65.

[12] Lowry, O.H., Rosenbury, N.J., Farr, A.L. and Randall, R.J. (1951). Protein measurement with folin phenol reagent. $J$. Bio.Chem., 193: 262-275.

[13] Bates, L.S., Waldren, R.P. and Teare, I.D. (1973). Rapid determination of the proline in water stress studies. Plant and Soil., 39: $205-208$. 
[14] Jaworski, E.G. (1971). Nitrate reductase assay in intact plant tissues. Biochem. Biophy. Res. Commun., 43: 1274-1279.

[15] Addy, S.K. and Goodman, R.N. (1972). Polyphenol oxidase and peroxidise activity in apple leaves inoculated with a virulent or avirulent strain for Erwinia amylovora. Indian Phytopath., 25: $575-579$.

[16] Kar, M. and Mishra, D. (1976). Catalase, peroxidase and polyphenol oxidase activities during rice leaf senescence. Plant Physiol., 57: 315- 319.

[17] Minakshi, D., Singh, A.K., Singh, V.P., Mishra, P.K. and Singh, S.K. (2012). Studies on different concentration of Lead $(\mathrm{Pb})$ and Cadmium $(\mathrm{Cd})$ on growth and accumulation in different parts of Tulsi (Ocimumtenuifolium) L. International Journal of Environmental Sciences., 2(3): 1733- 1741.

[18] Sevugaperumal, R., Selvaraj, K. and Ramasubramanian, V. (2012) Removal of aluminium by padina as bioadsorbant. International Journal of Biological \& Pharmaceutical Research., 3(4): 610-615.

[19] Mahapatra, M., Sabat, G., Patro, L., Padhy, R. and Mohanty, B.K. (2015). Studies of Aluminum $\left(\mathrm{Al}_{2} \mathrm{O}_{3}\right)$ Stress on morphology and pigments of Vigna radiata L. Int. J. Adv. Res. Biol. Sci. 2(12): 173-177.

[20] Shafiq, M., Kabir, M., Iqbal, M.Z. and Faroozi, Z.R. (2008). Reduction in germination and seedling growth of Thespesia populnea, caused by lead and cadmium treatments. Pak. J. Bot., 40(6): 2419 - 2426.

[21] Eun, S.O., Youn, H.S., Lee, Y. (2000). Lead disturbs microtubule organization in the root meristem of Zea mays. Physiol. Plant., 103: 695-702.

[22] Molas, J. (2002). Changes of chloroplast ultra-structure and total chlorophyll concentration in cabbage leaves caused by excess organic Ni (II) complexes. Environ.Exp.Bot., 47: 115 -126 .

[23] Boddi, B., Orarecz, A.R. and Lehoczki, E. (1995). Effect of Cadmium on organisation and photoreduction of protochlorophyllide in dark - grown leaves and etioplast inner membrane preparations of wheat. Photosynthetica., 31: 411 420 .

[24] John, R., Ahmad, P., Gadgil, K. and Sharma, S. (2009). Heavy metal toxicity: Effect on plant growth, biochemical parameters and metal accumulation by Brassica juncea $\mathrm{L}$. International Journal of Plant Production., 3(3): 65-76.

[25] Vinod, K.G., Awasthi. and Chauhan, P.K. (2012). Cu and Zn tolerance and responses of the Biochemical and Physiochemical system of Wheat. Journal of Stress Physiology \& Biochemistry., (3): 203-213.

[26] Gautam, S., Kannaujiya, P. and Srivastava, M.N. (2015). Growth and biochemical responses of spinach (Spinacea oleracea L.) grown in $\mathrm{Zn}$ contaminated soils.Int. J. Rec. Biotech., 3(1): 7-12.

[27] Zengin, F.K. and Munzuroglu, O. (2006). Toxic effects of cadmium $\left(\mathrm{Cd}^{++}\right)$on metabolism of sunflower (Helianthus annuus L.) seedlings. Acta Agricul. Scand., Sect. B, Plant Soil Sci., 56: 224-229.
[28] Elloumi, N., Ben, F., Rhouma, A., Ben, B., Mezghani, I. and Boukhris, M. (2007). Cadmium induced growth inhibition and alteration of biochemical parameters in almond seedlings grown in solution culture. Acta Physiol. Plant ., 29: $57-62$.

[29] Verma, S. and Dubey, R.S. (2001). Effect of heavy metal on soluble sugar and enzymes of their metabolism in rice. Biol. Plant., 44: 117 - 123.

[30] Sarma, H, Sarma, A. and Sarma, C.M. (2009). Physiological studies of some weeds grown under heavy metal and industrial effluent discharge zone of fertilizer factory. J. Ecol. Nat. Env.,1(7): 173 - 177.

[31] Aldesuquy, H.S. (1998). Effect of seawater salinity and gibberellic acid on abscisic acid, aminoacids and water use - efficiency by wheat plants. Agrochem., 42: $61-$ 69.

[32] Kahane, I. and Poljakoffmayber, A. (1968). Effect of substrate salinity on the ability for protein synthesis in pea roots. Plant. Physiol., 43: 1115-1119.

[33] Dubey, R.S. (1998). Nitrogen metabolism in plants under salt stress. In: Strategies for improving salt tolerance in higher plants. Eds. Jaiswal, P.K., Singh, R.P. and Gulati, A. Oxford and 1BH pub. Co. Pvt. Ltd. New Delhi.129 - 157.

[34] Orcutt, D.M. and Nilsen, E.T. (2000). The physiology of plants under stress. Wiley, New York. 683.

[35] Prasad, M.N.V. (1997). Trace metals. In: Plant Ecophysiology. Eds. Prasad, M.N.V., Willey, New York, 207 -249 .

[36] Tandon, P.K. and Srivastava, M. (2004). Effect of cadmium and nickel on metabolism during early stages of growth in gram (Cicer arietinum L.) seeds. Indian J. Agric. Biochem., 17: $31-34$.

[37] Stewart, G.R. and Lee J.A. (1974). The role of proline accumulation in higher plants. Planta., 120: $279-289$.

[38] Schat, H. Sharma, S.S. and Voojis, R. (1997). Heavy metal induces accumulation of proline in metal tolerant and non tolerant ecotypes of Silene Vulgaris. Plant. Physiol., 101: 477 -482 .

[39] Singh, T.N., Aspinall, D. Palegm L.S. and Boggess, S.F. (1973). Stress metabolism II. Changes in proline concentration in excised plant tissues. Aust. J. Biol. Sci., 26: $57-63$.

[40] Jayakumar, S. and Ramasubramanian, V. (2009). Bioremoval of chromium using seaweeds as biosorbents. J.Basic and App. Biol., 3(3 \& 4):121 - 128.

[41] Addy, S.K. and Goodman, R.N. (1972). Polyphenol oxidase and peroxidase activity in apple leaves inoculated with a virulent or avirulent strain for Erwinia amylovora. Indian Phytopath., 25: 575 - 579.

[42] Balasimha, D. (1982). Regulation of peroxidase in higher plants a review. Plant. Physiol. \& Biochem., 9: 130 - 143.

[43] Sharma, P. and Bhardwaj, R. (1982). Effect of 24 epi Blon seed germination, seedling growth and heavy metal uptake in Brassica juncea L. Gen. App. Plant Physiol., 32: 1 - 2. 